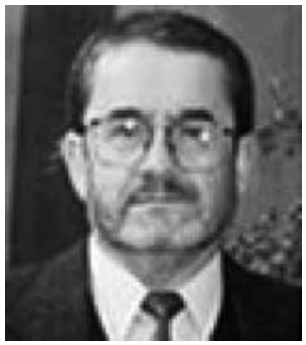

ULises VERGARA C. Director

\title{
EDITORIAL
}

\section{Readecuación de nuestro quehacer}

En Chile existe una creciente demanda de recursos humanos y profesionales de primer nivel que puedan responder con capacidad y liderazgo a las necesidades del país y de todas aquellas naciones que en virtud de los acuerdos comerciales y de cooperación internacional requieren de profesionales altamente calificados para enfrentar y resolver no sólo problemas individuales o poblacionales de salud animal y de diagnóstico y tratamiento veterinario, sino también para producir alimentos sanos y seguros, propender al desarrollo sustentable de los recursos silvoagropecuarios en un ambiente natural, asegurar el desarrollo humano y social de las comunidades rurales y resolver eventuales situaciones endémicas y emergentes, como consecuencia del aumento del riesgo sanitario que implica el proceso de globalización.

La Universidad de Chile en general y nuestra Facultad en particular han iniciado una readecuación de su quehacer con el propósito de responder eficiente y eficazmente a una realidad dinámica y cambiante que requiere de respuestas rápidas, oportunas y flexibles.

La misión de la Facultad se fundamenta en la creación, desarrollo y difusión de la Medicina Veterinaria, de las Ciencias Animales, de los Alimentos, Genómica y Salud Animal, Acuicultura, Biodiversidad y Protección y Conservación del medio ambiente, con el más alto nivel académico como aporte al desarrollo socioeconómico y científico del país.

Basado en ello, la Facultad debe entregar educación y formación superior, desarrollar investigación científica y tecnológica avanzada relevante y relacionada con la búsqueda de innovación y la exploración de nuevas áreas de interés nacional. Debe además transferir y difundir conocimientos, vinculándose con los diferentes agentes productivos, sociales y científicos.

En conformidad con lo anterior Avances en Ciencias Veterinarias, como revista de comunicación científica publicada por la Facultad de Ciencias Veterinarias y Pecuarias de la Universidad de Chile, debe adecuarse a las orientaciones de las nuevas autoridades de la Facultad y consolidarse como medio de expresión de la creatividad de los académicos y de los proyectos de investigación que se desarrollan en las distintas unidades universitarias y en otras instituciones del sector público o privado, interesadas en contribuir a:

- mantener la salud y bienestar de nuestros animales,

- proporcionar alimentos sanos y seguros a la población,

- mantener y mejorar el patrimonio sanitario del país,

- facilitar el crecimiento productivo en un marco de desarrollo sustentable,

- mantener la biodiversidad y estimular la protección y conservación del medio ambiente y

- el desarrollo equilibrado de las comunidades rurales a través de programas de capacitación y transferencia tecnológica.

Para cumplir este objetivo y facilitar la integración horizontal de los equipos de trabajo en esta nueva etapa de la institución, el Comité Editor de nuestra revista se ha conformado con académicos de la Facultad de Ciencias Veterinarias y Pecuarias, la Facultad de Ciencias Agronómicas y el Instituto de Nutrición y Tecnología de los Alimentos que, en conjunto con la Facultad de Ciencias Forestales, constituyen el Campus Sur de la Universidad de Chile. 